\title{
QT Prolongation and Torsades de Pointes Following Overdose of Ziprasidone and Amantadine
}

\author{
Alex F. Manini, MDa, Dara Raspberry, $\mathrm{MD}^{b}$, Robert S. Hoffman, MDa, Lewis S. Nelson, MD
}

aNew York City Poison Center, New York, NY

bJacobi Medical Center Emergency Medicine Residency Program, Bronx, NY

\section{INTRODUCTION}

The Food and Drug Administration (FDA) approved Ziprasidone, a second-generation atypical antipsychotic, in February 2001 [1]. Ziprasidone has a unique receptor profile that includes highaffinity antagonist activity at dopamine $\mathrm{D}_{2}$ receptors, inverse agonist activity at serotonin $(5-\mathrm{HT})_{2 \mathrm{~A}}$ receptors, agonist activity at $5-\mathrm{HT}_{1 \mathrm{~A}}$ receptors, and a relatively high affinity for serotonin and norepinephrine transporters [2]. It is recommended for the treatment of conditions that include, but are not limited to, acute mania, bipolar disorder, schizophrenia, and acute psychotic agitation [3-7].

Antipsychotic drug-induced QTc prolongation is of major concern because epidemiologic data and case-control studies have indicated an increased rate of sudden death in psychiatric patients taking antipsychotic drugs [8-11]. In 2002, just one year after FDA approval, a report issued from the FDA noted that safety studies comparing ziprasidone and other QT-prolonging drugs were lacking, and "an additive effect of ziprasidone and other drugs that prolong the QT interval cannot be excluded" [12]. Studies found that patients with dementia-related psychosis, treated with atypical antipsychotic medications, including ziprasidone, were at increased risk of death when compared to placebo treated patients. Following the findings of these studies, the FDA in 2005 recommended a black box warning [13]. Subsequently, a recommendation was placed in the product labeling that the agent should not be administered to patients who have significant cardiovascular illness or electrolyte imbalance or are receiving other agents that may prolong the QT interval [14].

Ziprasidone is widely reported to cause QTc prolongation, but without increased risk of Torsades de Pointes (TdP) [2,15-19]. However, very recent literature reports may suggest the possibil- ity of TdP [20,21]. This case report describes a 30-year-old woman who overdosed with ziprasidone, amantadine, and ibuprofen, and who displayed QT prolongation leading to TdP.

\section{CASE REPORT}

A 30-year-old woman was found unresponsive by a family member. The family reported that she was depressed ever since her father died 2 weeks previously, and 5 hours earlier that day she voiced a suicidal threat to her family. Emergency Medical Technicians found empty bottles of ziprasidone (60 mg tablets), amantadine (100 mg tablets), and ibuprofen (600 mg tablets) present on the floor near the patient. The family reported that she fell from her wheelchair to the ground and sustained minor trauma to the right leg.

Her past medical history was remarkable for mental retardation and depression with psychotic features. Her regular medications included ziprasidone (60 mg twice daily) and amantadine (100 mg twice daily).

On arrival to the Emergency Department, the patient's vital signs were: blood pressure, $118 / 76 \mathrm{mmHg}$; pulse rate, 60 beats/min; respiratory rate, 16 breaths/min; and oral temperature, $98.6^{\circ} \mathrm{F}$. She was lethargic but rousable to voice. Her pupils were equal, round, and reactive to light. The head, eyes, ears, nose, and throat examinations were unremarkable. She was in no respiratory distress and her breath sounds were bilaterally clear. Auscultation of heart tones revealed regular rate and rhythm without murmurs, rubs, or gallops. Abdominal examination revealed normal bowel sounds without tenderness or organomegaly. Skin examination was normal. Examination of the extremity revealed moderate ecchymosis of the right lower extremity overlying the popliteal fossa, but no deformity and $2+$ pulses distally. Neurological examination was not

Keywords: antipsychotics, torsades de pointes, toxicity

Notes: This case report was previously presented in abstract format at the $27^{\text {th }}$ International Congress of the European Association of Poison Centres and Clinical Toxicologists (Athens, Greece) in May 2007. There was no outside funding of any kind used for this study. Corresponding Author: Dr. Manini can be reached at 455 First Ave, Room 123, New York, NY. Email: manina01@med.nyu.edu 


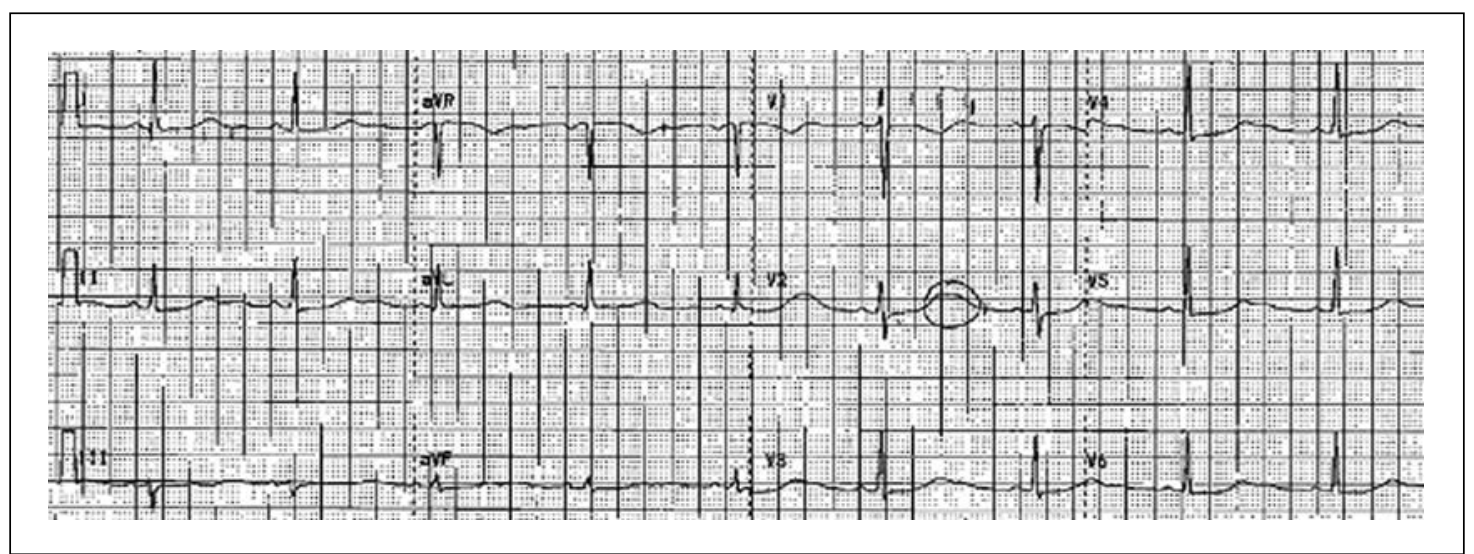

Figure 1: Initial electrocardiogram taken in the Emergency Department. In lead II, the QT is 800 ms, which combined with a pulse of 55/minute corresponds to a calculated QTc (Bazett) of $770 \mathrm{~ms}$.

completely assessed due to poor cooperation with the examination, but she was moving all extremities without a focal neurological deficit.

The patient was attached to a cardiac monitor and intravenous access was obtained. An electrocardiogram (Figure 1) performed on arrival to the Emergency Department and approximately 3 hours after her exposure, was notable for sinus bradycardia at 55 beats/min with a normal axis, QRS of 92 milliseconds (ms), and a markedly prolonged QT of $800 \mathrm{~ms}$ in lead II with a QTc (Bazett's correction) of $770 \mathrm{~ms}$ [22]. There was no terminal 40-ms right axis deviation of the QRS complex. We noted occasional premature ventricular contractions on the bedside cardiac monitor.

Laboratory evaluation was notable for a potassium of 3.0 $\mathrm{mEq} / \mathrm{L}$ and a magnesium of $1.9 \mathrm{mEq} / \mathrm{L}$. Results of bicarbonate, creatinine, complete blood count, calcium, cardiac biomarkers, and urinalysis were within normal limits. Serum acetaminophen, ethanol, salicylates, and a pregnancy test were all negative.

In the emergency department, she received one liter of normal saline intravenously, oral potassium replacement therapy, and was placed on 1:1 observation. The plan was to clear her medically and admit her to the psychiatric service. Approximately 8 hours after arrival to the ED and while waiting for an inpatient bed, the patient lost consciousness and showed a simultaneous appearance of a wide complex tachydysrhythmia on the monitor (Figure 2). The patient immediately received a succession of 5 defibrillations (monophasic defibrillator-100 J, $150 \mathrm{~J}, 200 \mathrm{~J}, 200 \mathrm{~J}, 200 \mathrm{~J}$ ) with subsequent return of spontaneous circulation and a normal sinus rhythm. The patient was endotracheally intubated without premedications during the course of the resuscitation. No chemical antidysrhythmics or magnesium replacement was administered prior to defibrillation and return of spontaneous circulation.

Post-resuscitation, she had a follow-up electrocardiogram that revealed a normal sinus rhythm with an excessively prolonged QT in lead II (720 ms) and a QTc (Bazett) that decreased to 750 $\mathrm{ms}$ (Figure 3). She received an intravenous magnesium bolus of 2 grams, followed by an infusion of $2.8 \mathrm{gm} /$ hour. She received an additional $40 \mathrm{mEq}$ intravenous potassium chloride over 1 hour

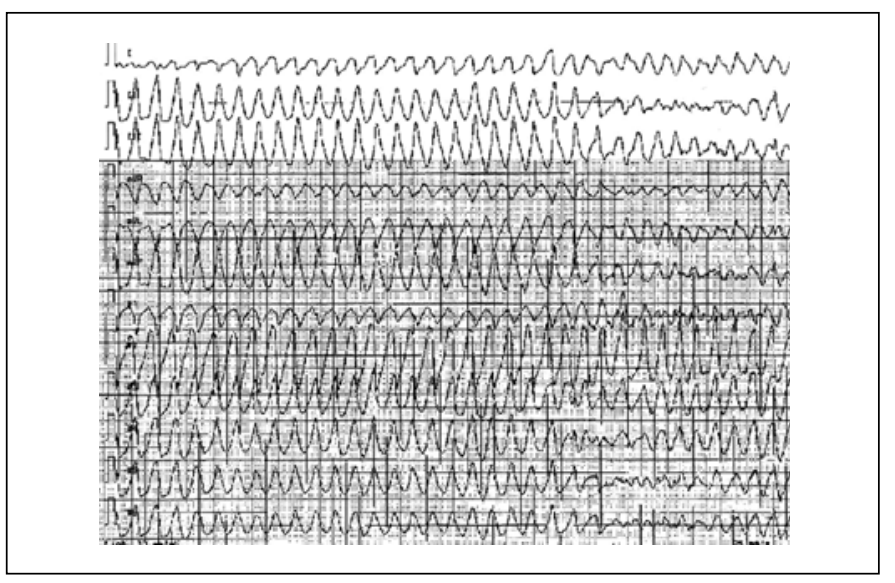

Figure 2: Electrocardiogram during cardiac arrest, taken approximately 8 hours after the patient's arrival to the Emergency Department. The torsades de pointes morphology is readily apparent.

and a total of $90 \mathrm{mEq}$ over the first 24 hours. Amiodarone 150 mg was intravenously loaded over 30 minutes. The patient was admitted to the Cardiac Care Unit (CCU) and the Poison Control Center was consulted.

After arrival in the CCU, amiodarone was discontinued. Her QTc remained approximately $540 \mathrm{~ms}$, and the subsequent 12 hours were complicated by episodes of sustained ventricular tachycardia (Figure 4). On CCU day 1, an isoproterenol infusion was initiated $(2 \mathrm{mcg} / \mathrm{min})$ in an attempt to achieve chemical overdrive pacing. No further episodes of TdP or ventricular tachycardia occurred once the QTc shortened below $500 \mathrm{~ms}$.

Her CCU course was complicated by bilateral pulmonary infiltrates on CCU day 3 and urosepsis initially detected from a urine specimen drawn on day 4 . Urine and blood cultures drawn on day 4 grew E. coli and her hemodynamics worsened, with three vasopressors required to maintain perfusion. Subsequently, she developed disseminated intravascular coagulation; and due to concerns 


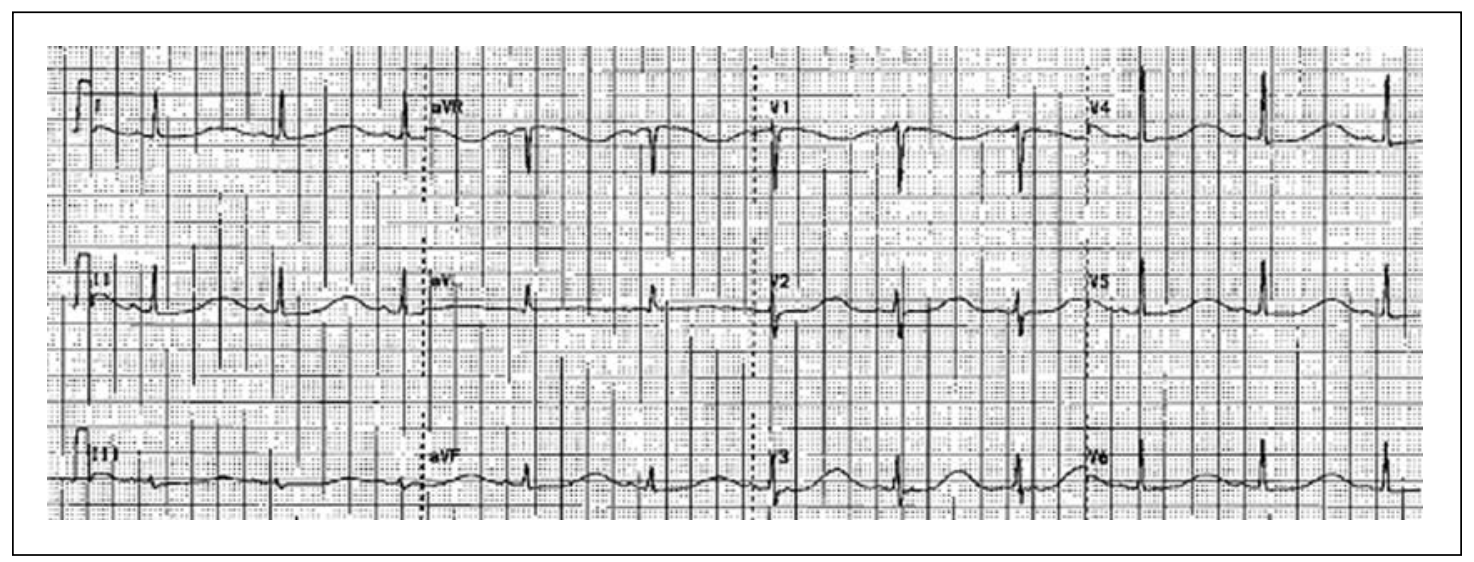

Figure 3: Electrocardiogram taken in the Cardiac Intensive Care Unit just 3 hours after torsades de pointes cardiac arrest. In lead II, the QT interval is $720 \mathrm{~ms}$, which combined with a pulse of 65/minute corresponds to a QTc (Bazett) of $750 \mathrm{~ms}$.

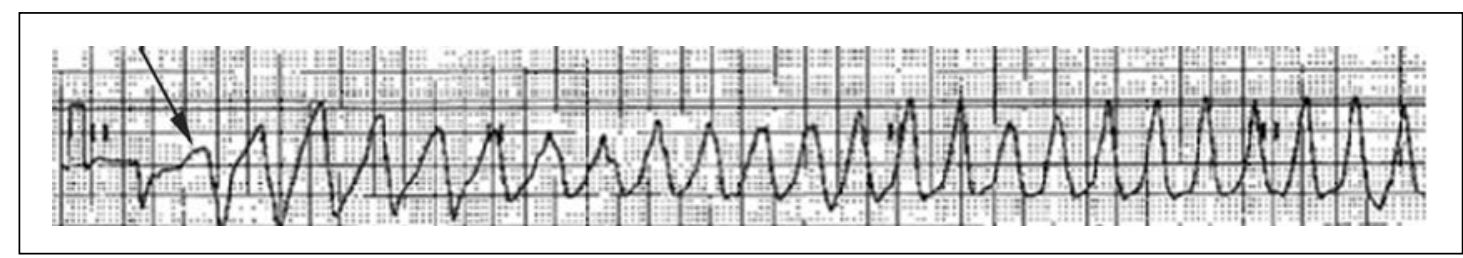

Figure 4: Rhythm strip taken in the Cardiac Intensive Care Unit following the initial resuscitation demonstrates one initial narrow QRS complex followed by a fusion beat (arrow) and subsequent sustained ventricular tachycardia.

of pulmonary embolism, computed tomography was performed with evidence of right lower extremity deep venous thrombosis as well as pulmonary embolus. She expired on CCU day 10. A postmortem revealed evidence of sepsis and a pulmonary embolism.

\section{DISCUSSION}

Given the history and clinical circumstances, we believe the episode of TdP was secondary to drug-induced QT prolongation. This history and findings at the scene were clearly consistent with overdose of her medications, which included ziprasidone and amantadine. Although we did not ascertain confirmatory serum levels, the patient openly admitted to suicidal overdose of her medications, and empty ziprasidone and amantadine bottles were found nearby at the scene. Following her initial cardiac arrest, she suffered numerous nosocomial complications including urosepsis, disseminated intravascular coagulation, and subsequent pulmonary embolism which together resulted in death.

Ziprasidone prolongs the QTc by blockade of potassium channels and subsequent interference with the rectifying potassium current $\left(\mathrm{I}_{K_{\mathrm{r}}}\right)$ [23-24]. In a study of 31 patients, ziprasidone, at a dose of $160 \mathrm{mg} /$ day, increased the mean QTc by $16 \mathrm{~ms}$ [25]. In placebo-controlled trials, ziprasidone, at $160 \mathrm{mg} /$ day, increased the mean QTc by $10 \mathrm{~ms}$ [26].
A meta-analysis from Justo and colleagues suggests that the most prevalent risk factors for development of psychotropic druginduced TdP include the female gender, presence of heart disease, hypokalemia, long QT syndrome, high dosing, and concomitant use of another QT-prolonging drug $[27,28]$. Of these risk factors, this patient had at least three, including amantadine co-ingestion and presence of hypokalemia [29-31]. However, the patient received oral potassium therapy hours before the occurrence of TdP; the only case report of amantadine-induced TdP was in a patient with a presentation of cardiac arrest early in the course of the overdose, with ventricular fibrillation being exacerbated, not improved, by administration of isoproterenol.

This patient had early signs (prolonged QT) but an 8-hour delay to occurrence of symptoms (TdP) during her Emergency Department course. When this period is added to the time from ingestion, this represents an important delay for initial symptoms of cardiac toxicity. In addition, recurrent episodes of sustained ventricular tachycardia over the first 12 hours in the CCU were unexpected since ziprasidone has a half-life of 4-10 hours and does not form any active metabolites [24]. In this case, an undescribed pharmacokinetic interaction between ziprasidone and amantadine cannot be ruled out as the cause of delayed dysrhythmias.

In conclusion, ziprasidone is currently regarded in the literature as a drug that does not induce TdP despite both an FDA-recommended black box warning and well-established 
prolongation of the QTc $[2,15-19,13,2,4,25,26]$. We reported the case of marked QT prolongation and TdP following overdose of ziprasidone and amantadine.

The authors have no potential financial conflicts of interest to report.

\section{REFERENCES}

1. FDA Drug Approvals - G. Available at http://www.fda.gov/ cder/approval/g.htm. Accessed September 27, 2006.

2. Nemeroff CB, Lieberman JA, Weiden PJ, et al. From clinical research to clinical practice: a 4-year review of ziprasidone. CNS Spectr. 2005;10(11):S1-20.

3. Perlis RH, Welge JA, Vornik LA, Hirschfeld RMA, Keck PE. Atypical antipsychotics in the treatment of mania: a metaanalysis of randomized, placebo-controlled trials. J Clin Psychiatry. 2006;67:509-516.

4. Patel NC, Keck PE. Ziprasidone: efficacy and safety in patients with bipolar disorder. Expert Rev Neurother. 2006;6 (8):1129-1138.

5. Stimmel GL, Gutierrez MA, Lee V. Ziprasidone: an atypical antipsychotic drug for the treatment of schizophrenia. Clin Ther. 2002;24:21-37.

6. Barak Y, Mazeh D, Plopski I, Baruch Y. Intramuscular ziprasidone treatment of acute psychotic agitation in elderly patients with schizophrenia. Am J Geriatr Psychiatry. 2006;14 (7):629-633.

7. Martel M, Sterzinger A, Miner J, Clinton J, Biros M. Management of acute undifferentiated agitation in the emergency department: a randomized double-blind trial of droperidol, ziprasidone, and midazolam. Acad Emerg Med. 2005;12 (12):1167-1172.

8. Ray WA, Meredith S, Thapa PB; Meador KG, Hall K, Murray KT. Antipsychotics and the risk of sudden cardiac death. Arch Gen Psychiatry. 1991;58:1161-1167.

9. Hennessy S, Bilker WB, Knauss JS, Margolis DJ, Kimmel SE, Reynolds RF, et al. Cardiac arrest and ventricular arrhythmia in patients taking antipsychotic drugs: cohort study using administrative data. BMJ. 2002;325:1070-1074.

10. Reilly JG, Ayis SA, Ferrier IN, Jones SJ, Thomas SHL. Thioridazine and sudden unexplained death in psychiatric inpatients. Br J Psychiatry. 2002;180:515-522.

11. Straus SM, Bleumink GS, Dieleman JP, van der Lei J, 't Jong GW, Kingma JH, et al. Antipsychotics and the risk of sudden cardiac death. Arch Intern Med. 2004;164:1293-1297.

12. 2002 Safety Alert—Geodon (ziprasidone $\mathrm{HCl}$ ). Available at: http://www.fda.gov/medwatch/SAFETY/2002/geodon.htm. Accessed October 13, 2006.

13. Alert for healthcare professionals_Ziprasidone (marketed as Geodon). Available at: http://www.fda.gov/cder/drug/ InfoSheets/HCP/ziprasidoneHCP.htm. Accessed September 27, 2006.
14. Geodon product information. Physicians' desk reference. 58th ed. Montvale, NJ: Thomson PDR, 2004:2597-2603.

15. Glassman AH, Bigger JT Jr. Antipsychotic drugs: prolonged QTc interval, torsade de pointes, and sudden death. Am J Psychiatry. 2001;158(11):1774-1782.

16. Kelly DL, Love RC. Ziprasidone and the QTc interval: pharmacokinetic and pharmacodynamic considerations. Psychopharmacol Bull. 2001 (Autumn);35(4):66-79.

17. Glassman AH. Schizophrenia, antipsychotic drugs, and cardiovascular disease. J Clin Psychiatry. 2005;66 Suppl 6:5-10.

18. Goodnick PJ. Ziprasidone: profile on safety. Expert Opin Pharmacother. 2001;2(10):1655-1662.

19. Citrome L, Volavka J. The promise of atypical antipsychotics: fewer side effects mean enhanced compliance and improved functioning. Postgrad Med. 2004;116(4):49-51,55-9,63.

20. Heinrich TW, Biblo LA, Schneider A. Torsades de pointes associated with ziprasidone. Psychosomatics. 2006;47(3):264-268.

21. Scahill L, Blair J, Leckman JF, Martin A. Sudden death in a patient with Tourette syndrome during a clinical trial of ziprasidone. J Psychopharmacol. 2005;19(2):205-206.

22. Bazett H. An analysis of the time relationship of electrocardiograms. Heart. 1920; 7:353-370.

23. Sanguinetti MC, Jiang C, Curran ME, Keating MT. A mechanistic link between an inherited and an acquired cardiac arrhythmia: HERG encodes the $\mathrm{I}_{\mathrm{kr}}$ potassium channel. Cell. 1995;81:299-307.

24. Juurlink D. Antipsychotics. In: Flomenbaum NE, Goldfrank LR, Hoffman RS, Howland MA, Lewin NA, Nelson LS (editors): Goldfrank's Toxicologic Emergencies. McGraw-Hill: $8^{\text {th }}$ ed., pp. 1040-1043.

25. Harrigan EP, Miceli JJ, Anziano R,Watsky E, Reeves KR, Cutler NR, et al. A randomized evaluation of the effects of six antipsychotic agents on QTc, in the absence and presence of metabolic inhibition. J Clin Psychopharmacol. 2004;24:62-69.

26. Citrome L, Volavka J, Czobor P, Brooks S, Loebel A, Mandel FS. Efficacy of ziprasidone against hostility in schizophrenia: Post hoc analysis of randomized, open-label study data. J Clin Psychiatry. 2006;67(4):638-642.

27. Justo D, Prokhorov V, Heller K, Zeltser D. Torsade de pointes induced by psychotropic drugs and the prevalence of its risk factors. Acta Psychiatr Scand. 2005:111:171-176.

28. Roden DM. Drug-induced prolongation of the QT interval. N Eng J Med. 2004;350:1013-1022.

29. Sartori M, Pratt CM, Young JB. Torsade de pointe. Malignant cardiac arrhythmia induced by amantadine poisoning. Am J Med. 1984;77(2):388-391.

30. Kay GN, Plumb VJ, Arciniegas JG, Henthorn RW, Waldo AL. Torsades de pointes: the long-short initiating sequence and other clinical features: observations in 32 patients. J Am Coll Cardiol. 1983;2:806-817.

31. Roden DM, Woosley RL, Primm RK. Incidence and clinical features of the quinidine-associated long QT syndrome: implications for patient care. Am Heart J. 1986;111:1088-1093. 